\title{
White-light oblique-incidence diffuse reflectance spectroscopy for classification of in-vivo pigmented skin lesions
}

Alejandro Garcia-Uribe, Elizabeth B. Smith, Madeleine Duvic, Lihong V. Wang

Alejandro Garcia-Uribe, Elizabeth B. Smith, Madeleine Duvic, Lihong V. Wang, "White-light oblique-incidence diffuse reflectance spectroscopy for classification of in-vivo pigmented skin lesions," Proc. SPIE 6435, Optical Interactions with Tissue and Cells XVIII, 64350L (21 February 2007); doi: $10.1117 / 12.699633$

SPIE. Event: SPIE BiOS, 2007, San Jose, California, United States 


\title{
White Light Oblique-Incidence Diffuse Reflectance Spectroscopy for Classification of In Vivo Pigmented Skin Lesions
}

\author{
Alejandro Garcia-Uribe ${ }^{1}$, Elizabeth B. Smith ${ }^{2}$, Madeleine Duvic ${ }^{3}$, Lihong V. Wang ${ }^{4}$ \\ ${ }^{1}$ Department of Electrical Engineering, Texas A\&M University, \\ 3128 TAMU, College Station, Texas, USA 77843-3128 \\ ${ }^{2}$ Department of Biomedical Engineering, Texas A\&M University \\ 3120 TAMU, College Station, Texas, USA 77843-3120 \\ ${ }^{3}$ Department of Dermatology, the University of Texas MD Anderson Cancer Center, 1515 Holcombe \\ Blvd., Houston, Texas, USA 77030 \\ ${ }^{4}$ Department of Biomedical Engineering, Washington University in St. Louis, \\ St. Louis, Missouri, USA 63130-4899
}

\begin{abstract}
A study of in-vivo classification of pigmented skin lesions using oblique-incidence diffuse reflectance spectroscopy is presented. Spatio-spectral data in the wavelength range from 455 to $765 \mathrm{~nm}$ are collected from 111 pigmented lesions including 10 histopathologically diagnosed as melanoma. The first 67 lesions are used for training the classifiers, and 44 lesions are used for testing. The first classifier separates (1) malignant melanoma and severe dysplastic nevi from (2) moderate and mild dysplastic nevi, common nevi, actinic and seborrheic keratoses. The second classifier next distinguishes between (a) moderate and mild dysplastic nevi, common nevi from (b) actinic and seborrheic keratoses. The third classifier further separates (I) moderate and mild dysplastic nevi from (II) common nevi. The first classifier performs with $100 \%$ sensitivity and $91 \%$ specificity with overall classification rates of $93 \%$ and $95 \%$ for the training and testing sets, respectively. The second classifier has classification rates of $95 \%$ and $97 \%$ for the training and testing sets, respectively, whereas the third classifier has classification rates of $98 \%$ and $94 \%$ for the training and testing sets, respectively.
\end{abstract}

Keywords: Oblique incidence reflectometry, Spectroscopy, Skin cancer, Lesion classification,

\section{INTRODUCTION}

Melanoma is the most serious and fatal type of skin cancer. The American Cancer Society estimates that there will be 62,000 new cases of melanoma in the U.S. this year with 8,000 people dying of the disease ${ }^{1}$. Several researchers have studied the potential use of spectroscopy for early detection of cutaneous malignant tumor. Wallace et al. ${ }^{2}$ used multivariate discriminate analysis and neural networks to classify reflectance spectra of melanoma and common nevi with an accuracy rate of $86.7 \%$. McIntosh et al. ${ }^{3}$ utilized near-infrared reflectance spectroscopy with multivariate analysis to group spectra from non-melanoma skin cancers, pre-cancerous, and benign lesions, with classification accuracies of $72.4-97.7 \%$. Tomatis et al. ${ }^{4}$ used multispectral imaging to discriminate between melanoma and non-melanoma lesions with $85 \%$ accuracy. Sigurdson et al. ${ }^{5}$ employed Raman spectroscopy to categorize skin lesions, including melanoma with a classification performance of $80.5 \%$. Garcia-Uribe et al. ${ }^{6}$ reported a $95 \%$ classification rate distinguishing dysplastic nevi from common nevi and a $100 \%$ classification rate separating carcinomas from keratosis.

\section{EXPERIMENTAL SETUP}

The schematic of the system is illustrated in Figure 1. White light from a halogen lamp is coupled to an optical fiber (200 $\mu \mathrm{m}$ diameter). When the probe is placed perpendicular to the area of interest, the center source fiber is normal to the surface of incidence. The fiber optic probe consists of five source fibers and 24 collection fibers. The outer source fibers are situated at 45 degrees and the remaining source fibers were placed at 25 degrees, Figure 2 . An optical multiplexer allows delivering light through only one source fiber at a time to the area of interest. Once the light is delivered to the skin, it interacts with the medium and the diffuse reflectance is collected by another set of optical fibers (100 $\mu \mathrm{m}$ diameter). The collection fibers are coupled with an imaging spectrograph (Newport, Oriel

Optical Interactions with Tissue and Cells XVIII, edited by Steven L. Jacques, William P. Roach, Proc. of SPIE Vol. 6435, 64350L, (2007) · 1605-7422/07/\$18 · doi: 10.1117/12.699633 
MS 257) that generates an optical spectrum for each fiber. A CCD camera (Andor 412DV) collects the spectralimages from the wavelength range 455 to $765 \mathrm{~nm}$. This information is stored on a computer for future data analysis.

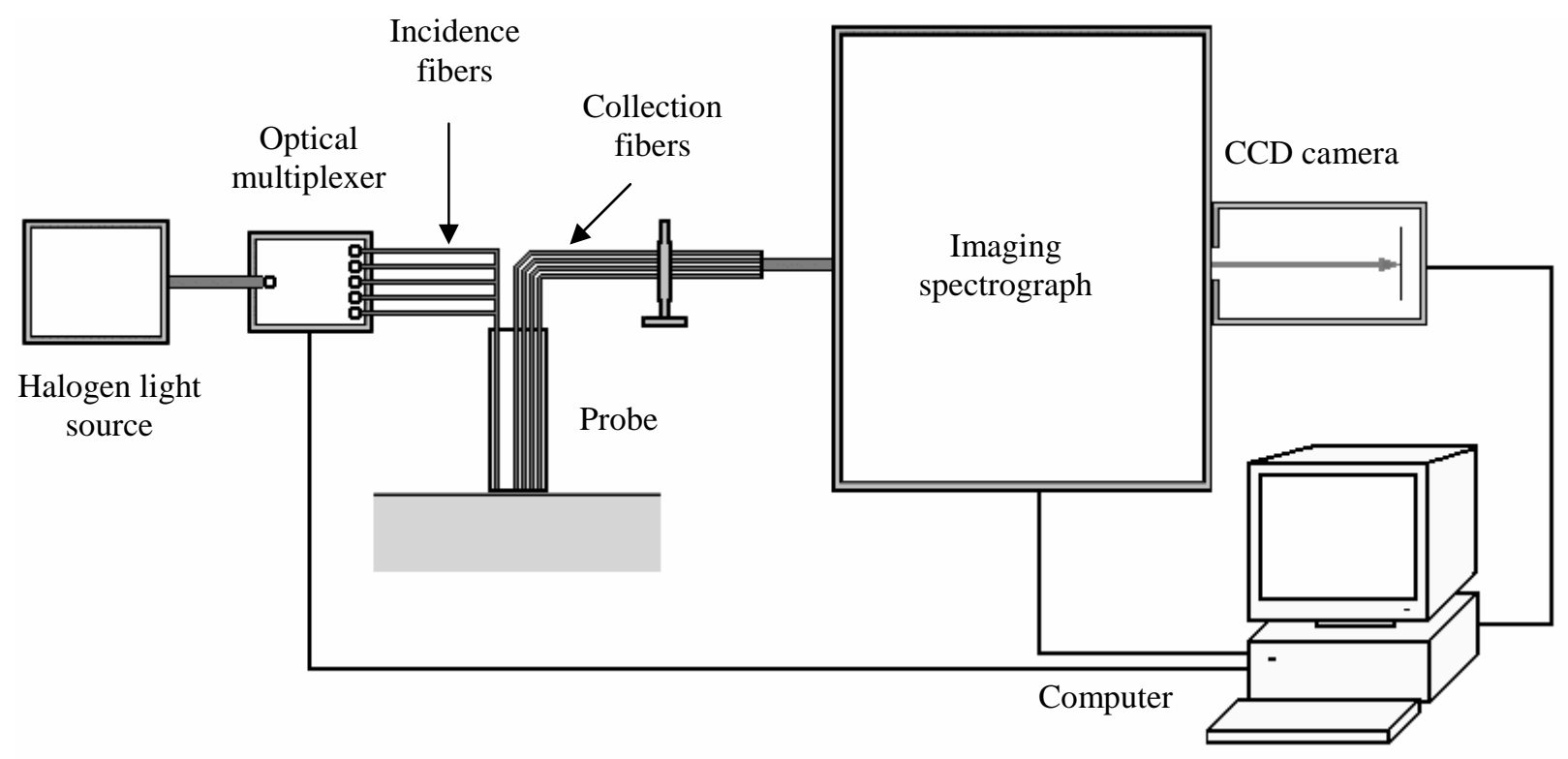

Figure 1: System Setup.

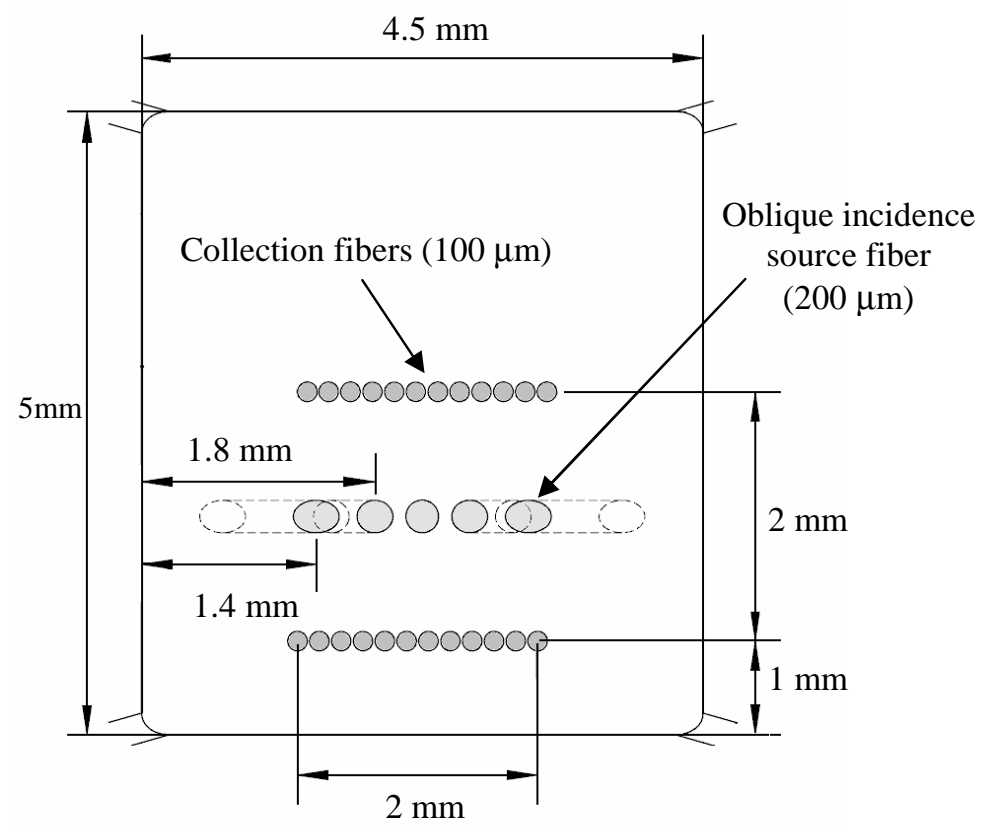

Figure 2: Probe description

\section{DATA DESCRIPTION}

Image acquisition was performed at The University of Texas M.D. Anderson Cancer Center (Melanoma and Skin Center). The oblique-incidence spectroscopic probe was used to collect spatio-spectral images from skin lesions and adjacent healthy skin. Data was collected with the probe placed perpendicular to the skin plane, ensuring contact but not compressing the skin. The spectral images were collected from five source fibers at four probe positions 
resulting in 20 images from each lesion and corresponding surrounding healthy tissue. And example of a spectral image is shown in figure 3.

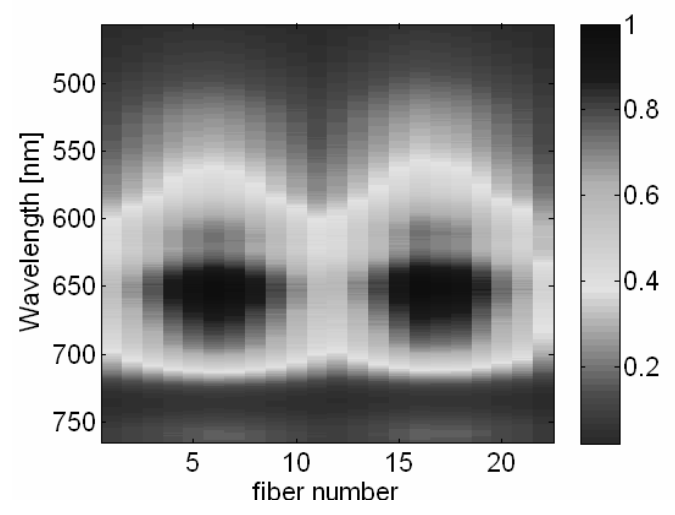

Figure 3. Spectral Image

The 111 pigmented lesions included in data analysis include benign common nevi (CN), mildly dysplastic nevi (DN1), moderately dysplastic nevi (DN2), severely dysplastic nevi (DN3), melanoma in situ (M1), invasive melanoma (M2), actinic keratosis (AK), seborrheic keratosis (SK). The number of lesions is described in table 1.

Table 1. Data description

\begin{tabular}{|lc|lc|lc|}
\hline Melanoma & 5 & $\begin{array}{l}\text { Mild Dysplastic } \\
\text { Nevi }\end{array}$ & 21 & Actinic Keratosis & 3 \\
\hline Melanoma in situ & 5 & $\begin{array}{l}\text { Moderate Dysplastic } \\
\text { Nevi }\end{array}$ & 41 & $\begin{array}{l}\text { Seborrheic } \\
\text { Keratosis }\end{array}$ & 12 \\
\hline $\begin{array}{l}\text { Severe Dysplastic } \\
\text { Nevi }\end{array}$ & 5 & Common Nevi & 19 & & \\
\hline
\end{tabular}

\section{DATA PROCESSING}

Each spectral image was normalized by the related healthy tissue image. This is based on the assumption that the melanin content of healthy skin tissue will correlate with that of the imaged lesion. The skin is not homogeneous and will vary within the collection site. To reduce the error, the collection fiber intensity found at each probe position was averaged for each source fiber. For simplification, groups were separated into two classes at a time. Features were extracted from the pigmented lesions to break them into two groups. This was repeated for the subgroups until the desired categories were achieved. Figure 4 illustrates the classification order followed for pigmented lesions.

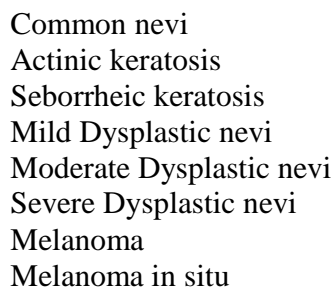

Melanoma

Melanoma in situ

Severe Dysplastic nevi

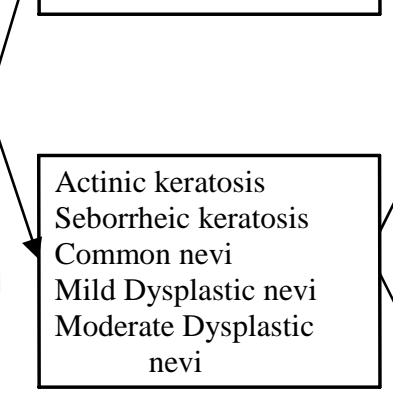

Common nevi

Mild Dysplastic nevi

Moderate Dysplastic nevi

Actinic keratosis

Seborrheic keratosis

Figure 4. Breakdown of pigmented lesions 
The continuous wavelet transform (CWT) was employed to extract the best five features per fiber in the two classes under analysis. The CWT of $f(t)$ with respect to the wavelet function $\Psi$ is defined as follows':

$$
W f_{\Psi}(b, a)=\frac{1}{\sqrt{a}} \int_{-\infty}^{\infty} f(t) \Psi\left(\frac{t-b}{a}\right) d t, a<0
$$

The parameter $\mathrm{b}$ is called the translation parameter. This parameter indicates the position or location of the wavelet function. The parameter $a$ is called the dilatation parameter. The inverse of this parameter $1 / a$ can be regarded as a measure of frequency. The one dimensional data, intensity as a function of wavelength, is converted into two dimensional information, wavelet transformation as a function of $b$ and $a$. CWT features are extracted from the two dimensional data yielding mean, standard deviation, skewness, and kurtosis values for varying wavelength ranges. The five most effective features for each fiber were chosen using the Fisher distance $(F D)$ as a measure of separability between classes. The Fisher distance is defined as $F D=\left|\mu_{1}-\mu_{2}\right| / \sqrt{\sigma_{1}{ }^{2}+\sigma_{2}{ }^{2}}$, where $\mu_{1}$ and $\mu_{2}$ denote class means and $\sigma_{1}$ and $\sigma_{2}$ denote class standard deviations. Redundant features were removed from the list and those remaining were narrowed down to the best twenty also using the Fisher distance. These twenty features form a feature vector that represents each lesion. Before feeding the feature vectors into a classifier the number of feature is optimized using a genetic algorithm as described in ref. 6 . The final feature vectors were processed to remove their correlations and reduce their dimensionality to one dimension. This final value represents each lesion and is called combined image feature CIF. The CIF values are user to design and test three statistical Bayes classifiers. ${ }^{6,8}$

The first classifier separates malignant melanoma, melanoma in situ and severe dysplastic nevi from actinic keratosis, seborrheic keratosis, common nevi, mild dysplastic nevi and moderate dysplastic nevi. This classifier performs with $100 \%$ sensitivity and $91 \%$ specificity. The classification rates are $93 \%$ and $95 \%$ for the training and testing sets, respectively.

Tanning set

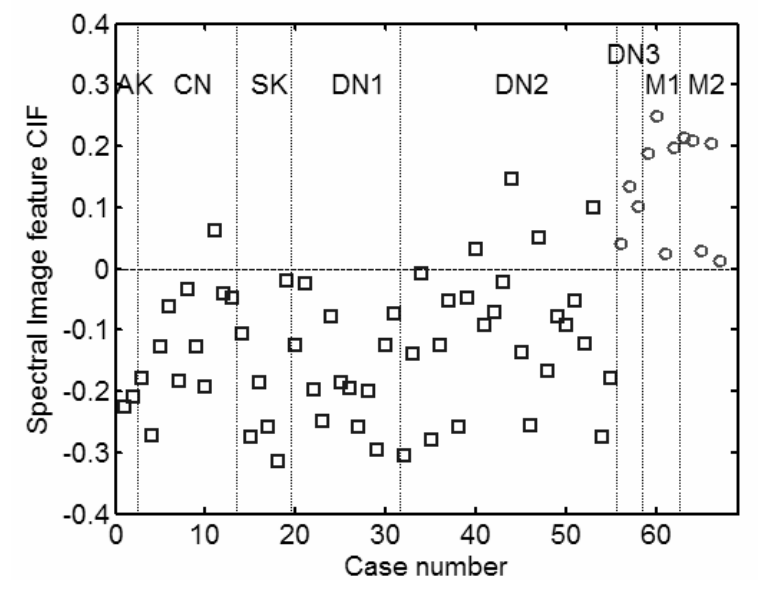

Testing set

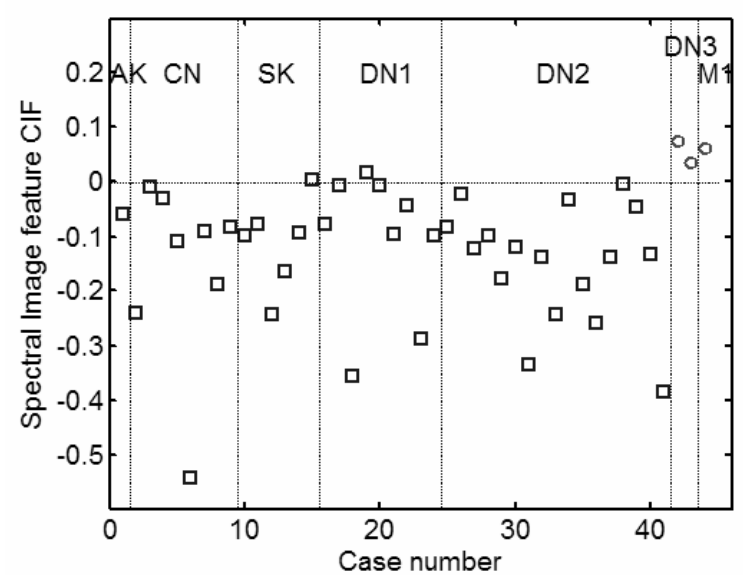

Figure 5. Scatter plot first classifier

The second classifier distinguishes actinic and seborrheic keratosis from common nevi, mild dysplastic nevi and moderate dysplastic nevi. This classifier uses fifty five lesions for training and forty one lesions for testing. The classification rate is $95 \%$ and $97 \%$ for the training and testing sets, respectively. 
Tanning set

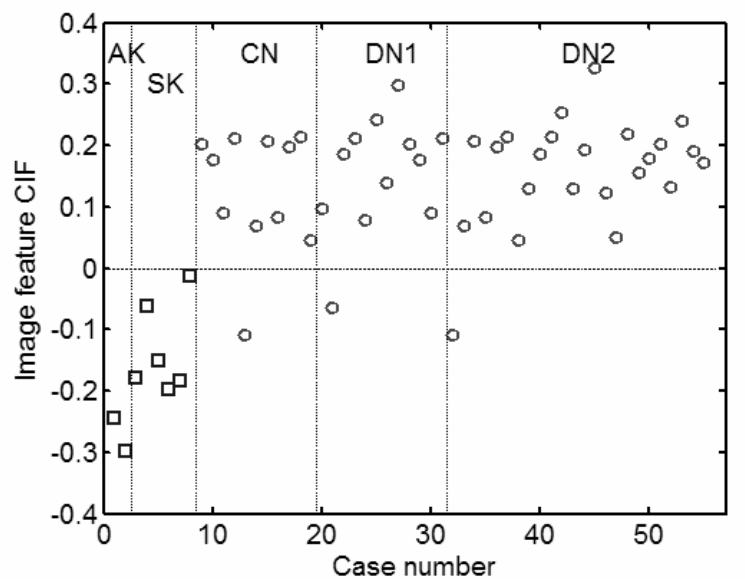

Testing set

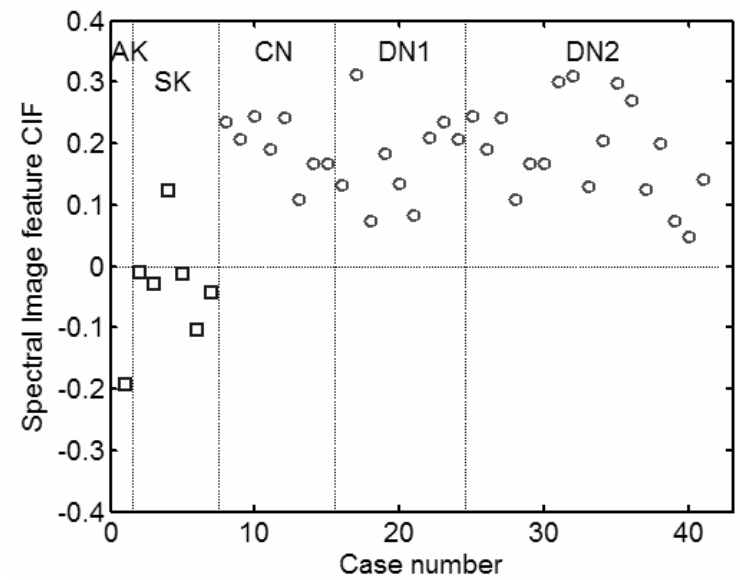

Figure 6. Scatter plot second classifier

Finally a third classifier is used to separate common nevi from mild and moderate dysplastic nevi. This classifier uses forty seven lesions for training and thirty one lesions for testing. The classification rate for this classifier is $98 \%$ and $94 \%$ for the training and testing sets, respectively. The Figure 5, 6 and 7 show the scatter plots for the training and testing sets for each classifier.

Training set

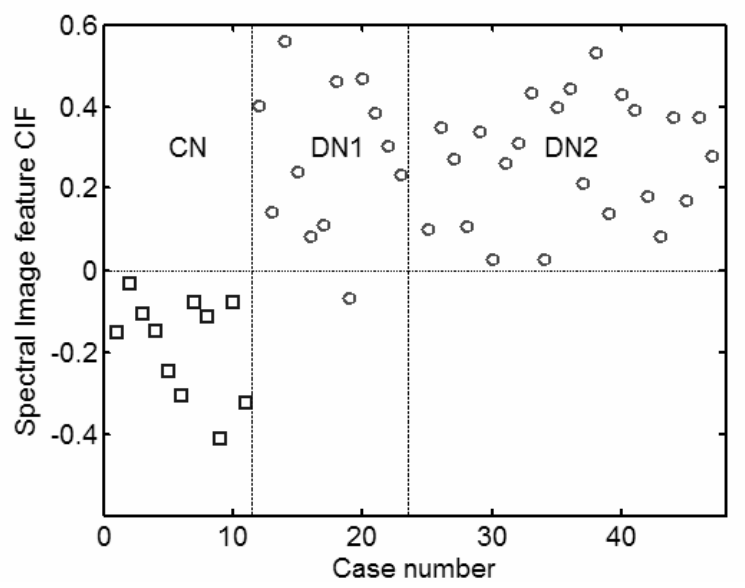

Testing set

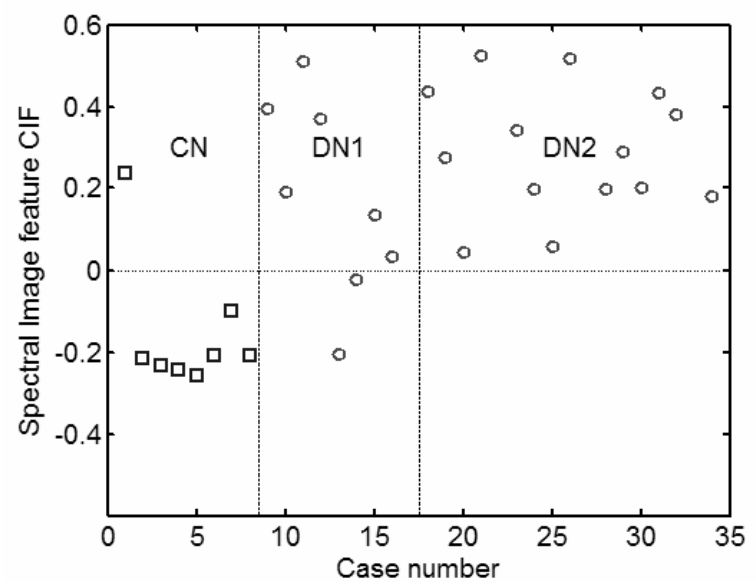

Figure 7. Scatter plot third classifier

The overall performance for the combination of the three classifiers is $91 \%$ and $87 \%$ for the tanning and testing sets respectively. The overall confusion matrices for the training and testing sets are presented in Tables 1 and 2.

Table 2. Confusion matrix for the training set

\begin{tabular}{|l|c|c|c|c|c|}
\hline & DN3, M1, M2 & AK, SK & CN & DN1, DN2 & Hit rate \\
\hline DN3, M1, M2 & 12 & & & & $100 \%$ \\
\hline AK, SK & & 8 & & & $100 \%$ \\
\hline CN & 1 & & 10 & & $91 \%$ \\
\hline DN1, DN2 & 4 & & 1 & 31 & $86 \%$ \\
\hline \multicolumn{7}{|l|}{} &
\end{tabular}


Table 3. Confusion matrix for the testing set

\begin{tabular}{|l|c|c|c|c|c|}
\hline & DN3, M1, M2 & AK, SK & CN & DN1, DN2 & Hit rate \\
\hline DN3, M1, M2 & 3 & & & & $100 \%$ \\
\hline AK, SK & & 5 & & 2 & $71 \%$ \\
\hline CN & 1 & 1 & 7 & & $88 \%$ \\
\hline DN1, DN2 & 1 & 1 & 23 & $88 \%$ \\
\hline \multicolumn{5}{l|}{}
\end{tabular}

\section{CONCLUSIONS}

This paper represents a study classifying pigmented skin lesions using oblique-incidence diffuse reflectance spectroscopy. The classifier that separates malignant from benign lesions achieves a sensitivity of $100 \%$ with a specificity of $91 \%$. The overall performance for the combination of the three classifiers is $91 \%$ and $87 \%$ for the tanning and testing sets respectively. The presented classification scheme does not require assumptions to produce a successful classification outcome. These results indicate that the approach presented can potentially be used by for computer-assisted classification technique of pigmented skin lesions.

\section{ACKNOWLEDGMENT}

Thanks to Dr. Mays, Dr. Hymens and Dr. Mansfield for their help during the data collection. This project is sponsored by NIH grant R01 CA106728. Wang's email is lhwang@ seas.wustl.edu.

1 American Cancer Society website. Melanoma and Non-melanoma Skin Cancer. http://www.cancer.org

2. V. P. Wallace, J. C. Bamber, D. C. Crawford, R. J. Ott, and P. S. Mortimer, "Classification of reflectance spectra from pigmented skin lesions, a comparison of multivariate discriminant analysis and artificial neural networks," Physics in Medicine and Biology 45, 2859-71 (2000).

3. L. M. McIntosh, R. Summers, M. Jackson, H. H. Mantsch, J. R. Mansfield, M. Howlett, A. N. Crowson, J. W. Toole, "Towards non-invasive screening of skin lesions by near-infrared spectroscopy", Journal of Investigative Dermatology 116 (1), 175-81 (2001).

4. S. Tomatis, M. Carrra, A. Bono, C. Bartoli, M. Lualdi, G. Tragni, A. Colombo, R. Marchesini, "Automated melanoma detection with a novel multispectral imaging system: results of a prospective study", Physics in Medicine and Biology 50, 1675-1687 (2005).

5. S. Sigurdsson, P. A. Philipsen, L. K. Hansen, J. Larsen, M. Gniadecka, and H. C. Wulf, "Detection of Skin Cancer by Classification of Raman Spectra", IEEE Transactions on Biomedical Engineering, no 10, vol. 51, Oct. (2004).

6 A. Garcia-Uribe, N. Kehtarnavaz, G. Marquez, V. Prieto, M. Duvic, and L. V. Wang, "Skin Cancer Detection by Spectroscopic Oblique-Incidence Reflectometry: Classification and Physiological Origins," Appl. Opt. 43, 2643-2650 (2004)

7. J. Groswami and A. Chan, Fundamentals of Wavelets, Theory, Algorithms and Application, 1st ed. Wiley Interscience, New York 1999.

8. R. O. Duda, P .E. Hart, D. G. Stork, Pattern Classification, Wiley Interscience Publication, second edition, New York 2000. 